\title{
Use and Safety of Prophylactic Endoscopy From a Single Center Serving Urban and Rural Children With Portal Hypertension
}

Voytek Slowik ( $\sim$ vslowik@cmh.edu )

Children's Mercy Hospital

Anissa Bernardez

University of Missouri-Kansas City

Heather Wasserkrug

Children's Mercy Hospital

Ryan T. Fischer

Children's Mercy Hospital

James F. Daniel

Children's Mercy Hospital

Tassos Grammatikopoulos

King's College Hospital NHS Foundation Trust

\section{Research Article}

Keywords: hepatology, varices, hemorrhage, gastrointestinal, pediatrics

Posted Date: May 27th, 2021

DOI: https://doi.org/10.21203/rs.3.rs-544137/v1

License: (1) This work is licensed under a Creative Commons Attribution 4.0 International License.

Read Full License

Version of Record: A version of this preprint was published at Scientific Reports on January 7th, 2022.

See the published version at https://doi.org/10.1038/s41598-021-03759-x. 


\section{Abstract}

Background: Prophylactic endoscopy is routine in adults with portal hypertension (PHTN), but there is limited data in pediatrics. We sought to describe our experience with prophylactic endoscopy in pediatric PHTN

Methods: Retrospective cohort study of 87 children who began surveillance endoscopy prior to gastrointestinal bleeding (primary prophylaxis) and 52 who began after an episode of bleeding (secondary prophylaxis) from 01/01/1994-07/01/2019.

Results: Patients who underwent primary prophylaxis had a lower mean number of endoscopies (3.897 vs $6.269, p=0.001)$. The primary prophylaxis group was less likely to require a portosystemic shunt $(6 \%$ vs $15 \%, p<0.001$ ) with no difference in immediate complications ( $1 \%$ vs $2 \%, p=0.173$ ), 2-week complications ( $1 \%$ vs $2 \%, p=0.097)$, need for transplant $(24 \%$ vs $27 \%, p=0.0819)$ or death $(5 \%$ vs $13 \%, p$ $=0.061)$. No deaths were related to variceal bleeding or endoscopy.

Conclusions: Primary and secondary endoscopic prophylaxis should be considered safe for the prevention of variceal hemorrhage in pediatric portal hypertension. There are differences in outcomes in primary and secondary prophylaxis, but unclear if this is due to patient characteristics versus treatment strategy. Further study is needed to compare safety and efficacy to watchful waiting.

\section{Introduction}

Portal hypertension (PHTN) is a well-established risk factor for gastrointestinal hemorrhage in pediatrics ${ }^{1}$. It is the result of obstructed blood flow through the portal venous system at the prehepatic, sinusoidal or post hepatic level. The underlying causes are variable and include portal vein thrombosis, mass effect, intrinsic liver disease and cirrhosis, among others. This increased resistance leads to an elevated portosystemic gradient (PSG) resulting in the development of varices as blood travels through shunts to bypass the obstructed portal system and return to venous circulation. While many complications can arise from PHTN, gastrointestinal hemorrhage secondary to varices requires emergent evaluation and treatment to prevent morbidity and mortality ${ }^{2}$.

However, the role of preventative management for esophageal varices does not have consensus between children and adults ${ }^{2,3}$. In adults with PHTN without an episode of bleeding, there are recommendations for primary preventative endoscopy to reduce the morbidity and mortality from variceal bleeding ${ }^{2}$. Endoscopy may lead to treatment decisions including use of non-selective beta blockers (NSBB), endoscopic variceal ligation (EVL), and portosystemic shunting. In pediatrics, there is a debate about the role of NSBB therapy and EVL in the prevention of bleeding complicated by a paucity of data ${ }^{3}$. There are multiple calls for further research to establish the safety and efficacy of prophylactic endoscopy and associated long term outcomes and create more objective guidance on treatment ${ }^{4,5}$. Indeed, some centers have already published data to challenge these recommendations including Duché et al ${ }^{6,7}$. 
Additionally, Molleston et al have recently published a 6 week mortality rate for variceal bleeding of $8.8 \%$ in the United States with Black or Hispanic children at higher risk ${ }^{8}$. While this rate is lower than in adults, it remains a significant risk to children and deserves further attention.

Our tertiary medical center serves a significant rural population who do not always have immediate access to appropriate medical care in the setting of variceal hemorrhage. With this population, we have historically adopted a primary prophylactic approach to endoscopy for variceal hemorrhage that has continued with some patients developing gastrointestinal hemorrhage prior to endoscopy and others more rarely choosing secondary prophylaxis. In this retrospective cohort study, our primary aim was to describe our experience and safety parameters with prophylactic endoscopy (as described in methods) to prevent gastrointestinal bleeding or recurrent gastrointestinal bleeding in pediatric PHTN. We also sought to describe outcomes in portosystemic shunt and transplant free survival. Lastly, we sought to determine if there were significant differences between our primary and secondary prophylactic endoscopy groups.

\section{Methods}

The Institutional Review Board at Children's Mercy - Kansas City approved all research activities and conformed ethical approval to the guidelines of the 1975 Declaration of Helsinki. Given the retrospective nature of the study, a waiver of consent was approved by the same board. Subjects were retrospectively identified using an internal electronic health record system database search. Patients met inclusion criteria if they had a diagnosis of PHTN and underwent initial endoscopy prior to age 18 years. Patients were grouped into primary prophylaxis if their initial endoscopy was for variceal screening due to the clinical diagnosis of hypersplenism or other manifestation of PHTN without history of gastrointestinal bleeding or anemia. Manifestations of PHTN included thrombocytopenia, ascites, radiography demonstrating signs of PHTN or other manifestation of PHTN. The secondary prophylaxis group included those with PHTN whose initial endoscopy at our center was to manage gastrointestinal bleeding or for surveillance after an episode of bleeding initially managed at an outside center. Some were from families who purposefully deferred endoscopy while the majority were those who developed bleeding prior to initial endoscopy. Hemoptysis and anemia (as indications for endoscopy) were also considered to be symptoms of gastrointestinal bleeding to avoid undercounting bleeding episodes. Clinical data was entered into a secured Research Electronic Data Capture database for further analysis. Data included age at time of endoscopy, underlying diagnosis, indication for endoscopy, endoscopic reports, and relevant clinical outcomes from January 01, 1994 to July 01, 2019. Pediatric End-Stage Liver Disease (PELD) and Model for End-Stage Liver Disease (MELD) scores were collected if available within 2 months of initial endoscopy excluding patients with cavernous transformation of the portal vein or portal vein thrombosis. If present, varices were described as grades $1-3$ based on currently accepted standards ${ }^{2,7}$.

\section{Institutional Endoscopic Protocol}

Patients with PHTN are clinically diagnosed at the Liver Care Center at Children's Mercy - Kansas City, using a combination of history, physical examination, laboratory, and radiographic findings by one of 
three board certified pediatric transplant hepatologists. The PSG is not routinely measured as its utilisation has not been validated in children. If PHTN is clinically suspected, patients are offered endoscopy for primary prophylaxis prior to an episode of variceal bleeding. At endoscopy, EVL is the treatment of choice for large esophageal varices, red wale signs, or with active hemorrhage for those where the banding apparatus can fit, typically after age 4 years or weight greater than 10 kilograms. Sclerotherapy is used if EVL is not feasible in the setting of active hemorrhage due to patient size, but is not routinely used in primary prophylaxis. Follow up endoscopy is repeated until varices are obliterated at an interval determined by the primary hepatologist based on presentation and severity, typically 2 weeks to 3 months. Afterwards, patients undergo surveillance endoscopy every 1-3 years with interval dependant on severity of varices, history of bleeding, progression of liver disease, and ability of family to travel to hospital. Those with refractory varices or recurrent bleeding are referred for portosystemic shunting or liver transplantation (LT). Patients who present initially with variceal bleeding receive similar secondary surveillance endoscopic schedule after control of bleeding and obliteration of varices. They are referred for portosystemic shunt or LT for the same indications.

\section{Statistical Analysis}

Patient and endoscopy characteristics were compared between the groups using t-tests and the MannWhitney U Test for continuous variables and chi-square or Fisher's exact tests for categorical variables. Kaplan-Meier survival curves and log rank tests were used assess overall survival between groups. To ensure that censuring is non-informative, Kaplan-Meier survival curves and log rank tests were also used to compare shunt and transplant free survival. The significance level was set at 0.05 and SPSS version 24 (IBM Corp, Armonk, NY) was used for all analyses. Given the exploratory nature of this study and the concern for type 2 errors, we intentionally did not correct for multiple statistical tests and felt that each individual finding was important.

\section{Results}

Of patients who underwent endoscopy and had a diagnosis of PHTN, we identified 87 subjects who underwent primary prophylaxis endoscopy and 52 in the secondary prophylaxis group. Patient level characteristics are reported in Table 1. There were no significant differences in gender or racial / ethnic background. For primary etiology, patients with autoimmune hepatitis and cavernous transformation / thrombosis of the portal vein groups had significant differences between groups. Both groups presented at similar ages for their initial endoscopy. There was no difference in initial MELD score between both groups while initial PELD score was found to have a statistically significant difference (1.92 vs 6.10, $p=$ 0.041) (Table 1). Mean number of years follow-up for all patients from first to last endoscopy was 3.21 years with a wide range ( $0-18.56$ years, IQR 5.35 years) (Fig. $1 \mathrm{a}$ and $1 \mathrm{~b})$. 
Table 1

Patient level characteristics of primary and secondary prophylactic endoscopy, $\mathrm{p}$-value $<0.05$ bolded.

CTPV - Cavernous Transformation of the Portal Vein, PVT - Portal Vein Thrombosis

\begin{tabular}{|c|c|c|c|c|c|}
\hline & \multicolumn{2}{|c|}{$\begin{array}{l}\text { Primary } \\
\text { Prophylaxis }\end{array}$} & \multicolumn{2}{|c|}{$\begin{array}{l}\text { Secondary } \\
\text { Prophylaxis }\end{array}$} & \multirow[b]{2}{*}{$\begin{array}{l}\mathrm{p}- \\
\text { value }\end{array}$} \\
\hline & $\mathbf{n}$ & $\%$ & $\mathrm{n}$ & $\%$ & \\
\hline Total patients & 87 & 1 & 52 & 1 & - \\
\hline Race / Ethnicity & - & - & - & - & - \\
\hline American Indian / Alaskan Native & 1 & 11 & 0 & 0 & $\begin{array}{l}p= \\
0.626\end{array}$ \\
\hline Asian & 3 & 3 & 1 & 2 & $\begin{array}{l}\mathrm{p}= \\
0.520\end{array}$ \\
\hline African American & 6 & 7 & 1 & 2 & $\begin{array}{l}p= \\
0.188\end{array}$ \\
\hline Caucasian & 70 & 80 & 44 & 85 & $\begin{array}{l}\mathrm{p}= \\
0.697\end{array}$ \\
\hline Hispanic / Latino & 4 & 5 & 4 & 8 & $\begin{array}{l}p= \\
0.343\end{array}$ \\
\hline More than one race reported & 2 & 2 & 0 & 0 & $\begin{array}{l}\mathrm{p}= \\
0.390\end{array}$ \\
\hline Pacific Islander & 0 & 0 & 1 & 2 & $\begin{array}{l}p= \\
0.374\end{array}$ \\
\hline Unknown / not documented & 1 & 1 & 1 & 2 & $\begin{array}{l}p= \\
0.610\end{array}$ \\
\hline Female (\%) & 48 & 55 & 30 & 58 & $\begin{array}{l}\mathrm{p}= \\
0.910\end{array}$ \\
\hline Underlying Diagnosis & - & - & - & - & - \\
\hline Alagille Syndrome & 5 & 6 & 1 & 2 & $\begin{array}{l}\mathrm{p}= \\
0.270\end{array}$ \\
\hline Alpha-1-antitrypsin Deficiency & 5 & 6 & 2 & 4 & $\begin{array}{l}\mathrm{p}= \\
0.475\end{array}$ \\
\hline Autoimmune Hepatitis & 8 & 9 & 0 & 0 & $\begin{array}{l}p= \\
0.021\end{array}$ \\
\hline Biliary Atresia & 14 & 16 & 16 & 31 & $\begin{array}{l}p= \\
0.068\end{array}$ \\
\hline Budd Chiari & 0 & 0 & 1 & 2 & $\begin{array}{l}\mathrm{p}= \\
0.374\end{array}$ \\
\hline
\end{tabular}




\begin{tabular}{|c|c|c|c|c|c|}
\hline \multirow[b]{2}{*}{ CTPV / PVT } & \multicolumn{2}{|c|}{$\begin{array}{l}\text { Primary } \\
\text { Prophylaxis }\end{array}$} & \multicolumn{3}{|c|}{$\begin{array}{l}\text { Secondary } \\
\text { Prophylaxis }\end{array}$} \\
\hline & 10 & 11 & 14 & 27 & $\begin{array}{l}p= \\
0.036\end{array}$ \\
\hline Chronic Heart Disease & 2 & 2 & 2 & 4 & $\begin{array}{l}p= \\
0.480\end{array}$ \\
\hline Congenital Hepatic Fibrosis & 8 & 9 & 3 & 6 & $\begin{array}{l}p= \\
0.353\end{array}$ \\
\hline Cystic Fibrosis Related Liver Disease & 13 & 15 & 4 & 8 & $\begin{array}{l}p= \\
0.160\end{array}$ \\
\hline Cystinosis & 0 & 0 & 1 & 2 & $\begin{array}{l}p= \\
0.374\end{array}$ \\
\hline Glycogen Storage Disease & 1 & 1 & 0 & 0 & $\begin{array}{l}p= \\
0.626\end{array}$ \\
\hline Joubert Syndrome & 1 & 1 & 0 & 0 & $\begin{array}{l}p= \\
0.626\end{array}$ \\
\hline Primary Sclerosing Cholangitis & 5 & 6 & 1 & 2 & $\begin{array}{l}p= \\
0.270\end{array}$ \\
\hline VOD/SOS & 1 & 1 & 0 & 0 & $\begin{array}{l}p= \\
0.626\end{array}$ \\
\hline Wilson's Disease & 2 & 2 & 0 & 0 & $\begin{array}{l}p= \\
0.390\end{array}$ \\
\hline Other (free text box) & 12 & 14 & 7 & 13 & $\begin{array}{l}p= \\
1.000\end{array}$ \\
\hline Mean age at first endoscopy, years (SD) & $\begin{array}{l}8.54 \\
(4.97)\end{array}$ & - & $\begin{array}{l}7.04 \\
(5.96)\end{array}$ & - & $\begin{array}{l}p= \\
0.131\end{array}$ \\
\hline Age range at first endoscopy, years & $\begin{array}{l}0.56- \\
17.87\end{array}$ & - & $\begin{array}{l}0.50- \\
17.88\end{array}$ & - & - \\
\hline $\begin{array}{l}\text { \# of patients with an available initial PELD score } \\
\text { (excluding CTPV and PVT) }\end{array}$ & 46 & 53 & 21 & 40 & - \\
\hline Mean PELD score (SD) & $\begin{array}{l}1.92 \\
(4.59)\end{array}$ & - & $\begin{array}{l}6.10 \\
(8.37)\end{array}$ & - & $\begin{array}{l}p= \\
0.041\end{array}$ \\
\hline Range of PELD score & $0-15.6$ & - & $0-28.8$ & - & - \\
\hline $\begin{array}{l}\text { \# of patients with an available initial MELD score } \\
\text { (excluding CTPV and PVT) }\end{array}$ & 18 & 21 & 5 & 10 & - \\
\hline Mean MELD score (SD) & $\begin{array}{l}10.56 \\
(5.85)\end{array}$ & - & $\begin{array}{l}12.00 \\
(3.16)\end{array}$ & - & $\begin{array}{l}p= \\
0.605\end{array}$ \\
\hline Range of MELD score & $6-26$ & - & $9-17$ & - & - \\
\hline
\end{tabular}




\begin{tabular}{|c|c|c|c|c|c|}
\hline \multirow[b]{2}{*}{ Mean of total endoscopic procedures (SD) } & \multicolumn{2}{|c|}{$\begin{array}{l}\text { Primary } \\
\text { Prophylaxis }\end{array}$} & \multicolumn{3}{|c|}{$\begin{array}{l}\text { Secondary } \\
\text { Prophylaxis }\end{array}$} \\
\hline & $\begin{array}{l}3.90 \\
(2.75)\end{array}$ & - & $\begin{array}{l}6.27 \\
(4.63)\end{array}$ & - & $\begin{array}{l}p= \\
0.001\end{array}$ \\
\hline Range of total endoscopic procedures & $1-13$ & - & $1-19$ & - & - \\
\hline Eradication of varices at final endoscopy & 6 & 7 & 8 & 15 & $\begin{array}{l}p= \\
0.188\end{array}$ \\
\hline $\begin{array}{l}\text { Number of patients who had bleeding despite primary } \\
\text { prophylaxis }\end{array}$ & 18 & 21 & - & - & - \\
\hline Shunt & 5 & 6 & 18 & 35 & $\begin{array}{l}p< \\
0.001\end{array}$ \\
\hline Meso-Rex Shunt & 0 & 0 & 4 & 8 & - \\
\hline Splenorenal Shunt & 5 & 6 & 13 & 25 & - \\
\hline Mesocaval Shunt & 0 & 0 & 1 & 2 & - \\
\hline Shunts who were eventually transplanted & 1 & 1 & 2 & 4 & - \\
\hline Mean number of endoscopies prior to shunt (SD) & $\begin{array}{l}5.6 \\
(3.65)\end{array}$ & - & $\begin{array}{l}4.61 \\
(3.94)\end{array}$ & - & - \\
\hline Liver transplants & 21 & 24 & 14 & 27 & $\begin{array}{l}p= \\
0.082\end{array}$ \\
\hline Death & 4 & 5 & 7 & 13 & $\begin{array}{l}p= \\
0.061\end{array}$ \\
\hline $\begin{array}{l}\text { Was cause of death related to variceal complications } \\
\text { or endoscopy }\end{array}$ & 0 & 0 & 0 & 0 & - \\
\hline
\end{tabular}

Endoscopic level characteristics can be found in Table 2. Indication for endoscopy was not included in statistical analysis as indications were part of the inclusion criteria for primary and secondary prophylaxis. However, if there was breakthrough GI bleeding, primary prophylaxis was associated with a lower number of hematemesis episodes ( $24 \%$ vs $51 \%$ of endoscopy for $\mathrm{GI}$ bleeding, $\mathrm{p}=0.02$ ). Primary prophylaxis was associated with significantly fewer admissions to the pediatric intensive care unit (PICU) at the time of endoscopy and lower graded esophageal varices. Additionally, secondary prophylaxis was associated with the patient having an active prescription for propranolol at the time of endoscopy (7\% vs $15 \%, p<0.001)$ and with higher dosing ( $0.86 \mathrm{vs} 1.11 \mathrm{mg} / \mathrm{kg} /$ day, $\mathrm{p}=0.035)$. There were no differences in the number of immediate and 2-week complications between groups. One patient treated with EVL for primary prophylaxis developed a partial esophageal stricture after 3 endoscopies. That patient required one-time balloon dilation without further management. A stricture in the secondary prophylaxis group, again after 3 endoscopies, did not allow the banding apparatus to pass preventing further treatment. 
That patient was referred for portosystemic shunting. The remaining complications, such as chest pain and sore throat, were successfully managed with supportive care (Table 2). 
Table 2

Endoscopy level characteristics of primary and secondary prophylactic endoscopy, $\mathrm{p}$-value $<0.05$ bolded

\begin{tabular}{|c|c|c|c|c|c|}
\hline & \multicolumn{2}{|c|}{$\begin{array}{l}\text { Primary } \\
\text { Prophylaxis }\end{array}$} & \multicolumn{2}{|c|}{$\begin{array}{l}\text { Secondary } \\
\text { Prophylaxis }\end{array}$} & \multirow[b]{2}{*}{$\begin{array}{l}\mathrm{p}- \\
\text { value }\end{array}$} \\
\hline & $\mathbf{n}$ & $\%$ & $\mathbf{n}$ & $\%$ & \\
\hline Total number of endoscopies & 339 & 100 & 326 & 100 & - \\
\hline Indication for endoscopy & - & - & - & - & - \\
\hline Initial endoscopy for primary prophylaxis & 76 & 22 & 0 & 0 & - \\
\hline Surveillance for primary prophylaxis & 198 & 58 & 0 & 0 & - \\
\hline Management of active GI bleeding & 29 & 9 & 90 & 28 & - \\
\hline $\begin{array}{l}\text { Follow up after control of bleeding for eradication of } \\
\text { varices }\end{array}$ & 7 & 2 & 69 & 21 & - \\
\hline $\begin{array}{l}\text { Surveillance for secondary prophylaxis (after } \\
\text { breakthrough bleeding in primary group) }\end{array}$ & 25 & 7 & 155 & 48 & - \\
\hline Anemia & 3 & 1 & 4 & 1 & - \\
\hline Other & 1 & $<1$ & 8 & 2 & - \\
\hline If GI bleed, presenting symptom & - & - & - & - & - \\
\hline Hematemesis & 7 & 24 & 46 & 51 & $\begin{array}{l}p= \\
0.020\end{array}$ \\
\hline Melena / bloody stools & 16 & 55 & 30 & 33 & $\begin{array}{l}p= \\
0.060\end{array}$ \\
\hline Anemia & 1 & 3 & 0 & 0 & $\begin{array}{l}p= \\
0.549\end{array}$ \\
\hline Hemoptysis & 2 & 7 & 3 & 3 & $\begin{array}{l}\mathrm{p}= \\
0.090\end{array}$ \\
\hline Other & 3 & 10 & 11 & 12 & $\begin{array}{l}p= \\
1.000\end{array}$ \\
\hline Admit location of endoscopy & - & - & - & - & - \\
\hline Same Day Surgery / Out-patient & 228 & 57 & 200 & 61 & $\begin{array}{l}p= \\
0.144\end{array}$ \\
\hline Floor & 106 & 27 & 99 & 30 & $\begin{array}{l}\mathrm{p}= \\
0.889\end{array}$ \\
\hline PICU & 4 & 1 & 24 & 7 & $\begin{array}{l}p< \\
0.001\end{array}$ \\
\hline
\end{tabular}




\begin{tabular}{|c|c|c|c|c|c|}
\hline \multirow[b]{2}{*}{ ED } & \multicolumn{2}{|c|}{$\begin{array}{l}\text { Primary } \\
\text { Prophylaxis }\end{array}$} & \multicolumn{2}{|c|}{$\begin{array}{l}\text { Secondary } \\
\text { Prophylaxis }\end{array}$} & \multirow[b]{2}{*}{$\begin{array}{l}p= \\
0.489\end{array}$} \\
\hline & 0 & 0 & 1 & $<1$ & \\
\hline Remaining is missing / unknown from EMR & 1 & $<1$ & 2 & $<1$ & - \\
\hline Variceal grade / size & - & - & - & - & $\begin{array}{l}p< \\
0.001\end{array}$ \\
\hline None & 49 & 14 & 37 & 11 & - \\
\hline Grade 1 / small / mild & 128 & 38 & 83 & 25 & - \\
\hline Grade 2 / medium / moderate & 75 & 22 & 28 & 9 & - \\
\hline Grade 3 / large / severe & 56 & 17 & 115 & 35 & - \\
\hline Present, but not graded & 31 & 9 & 63 & 19 & - \\
\hline Red wale sign & 74 & 22 & 82 & 25 & $\begin{array}{l}p= \\
0.291\end{array}$ \\
\hline Bleeds with red wale sign & 8 & 28 & 21 & 23 & $\begin{array}{l}p= \\
0.017\end{array}$ \\
\hline Gastric varices & 74 & 22 & 108 & 33 & $\begin{array}{l}p= \\
0.001\end{array}$ \\
\hline Bleeds with gastric varices & 3 & 10 & 29 & 32 & $\begin{array}{l}p< \\
0.001\end{array}$ \\
\hline Duodenal varices & 9 & 3 & 2 & $<1$ & $\begin{array}{l}p= \\
0.080\end{array}$ \\
\hline Bleeds with duodenal varices & 2 & 7 & 0 & 0 & $\begin{array}{l}p= \\
0.259\end{array}$ \\
\hline Portal hypertensive gastropathy & 132 & 39 & 144 & 44 & $\begin{array}{l}p= \\
0.232\end{array}$ \\
\hline Bleeds with portal hypertensive gastropathy & 12 & 41 & 39 & 43 & $\begin{array}{l}p< \\
0.001\end{array}$ \\
\hline Treatment of varices & - & - & - & - & - \\
\hline None & 201 & 69 & 134 & 46 & $\begin{array}{l}p< \\
0.001\end{array}$ \\
\hline Primary ligation & 105 & 36 & - & - & - \\
\hline Secondary ligation & 24 & 8 & 146 & 51 & $\begin{array}{l}p< \\
0.001\end{array}$ \\
\hline Primary sclerotherapy & 0 & 0 & - & - & - \\
\hline
\end{tabular}




\begin{tabular}{|c|c|c|c|c|c|}
\hline \multirow[b]{2}{*}{ Secondary sclerotherapy } & \multicolumn{2}{|c|}{$\begin{array}{l}\text { Primary } \\
\text { Prophylaxis }\end{array}$} & \multicolumn{2}{|c|}{$\begin{array}{l}\text { Secondary } \\
\text { Prophylaxis }\end{array}$} & \multirow[b]{2}{*}{$\begin{array}{l}p< \\
0.001\end{array}$} \\
\hline & 1 & $<1$ & 32 & 11 & \\
\hline Attempted but unsuccessful ligation & 8 & 3 & 14 & 5 & $\begin{array}{l}p= \\
0.239\end{array}$ \\
\hline Propranolol usage at the time of endoscopy & 23 & 7 & 50 & 15 & $\begin{array}{l}p< \\
0.001\end{array}$ \\
\hline Mean NSBB dose, mg/kg/day (SD) & $\begin{array}{l}0.86 \\
(0.39)\end{array}$ & - & $\begin{array}{l}1.11 \\
(0.59)\end{array}$ & - & $\begin{array}{l}p= \\
0.035\end{array}$ \\
\hline Range of NSBB dose, $\mathrm{mg} / \mathrm{kg} / \mathrm{day}$ & $\begin{array}{l}0.42- \\
1.64\end{array}$ & - & $\begin{array}{l}0.26- \\
2.53\end{array}$ & - & - \\
\hline How many bleeds occurred while on beta blocker & 3 & 13 & 6 & 12 & $\begin{array}{l}p= \\
0.233\end{array}$ \\
\hline Octreotide used prior to endoscopy & 7 & 2 & 15 & 5 & $\begin{array}{l}p= \\
0.099\end{array}$ \\
\hline Complications, immediate & 4 & 1 & 8 & 2 & $\begin{array}{l}p= \\
0.173\end{array}$ \\
\hline Bleeding during procedure & 1 & $<1$ & 6 & 2 & - \\
\hline Esophageal stricture & 1 & $<1$ & 1 & $<1$ & - \\
\hline Desaturations / respiratory distress & 2 & $<1$ & 1 & $<1$ & - \\
\hline Complications within 2 weeks & 3 & $<1$ & 8 & 2 & $\begin{array}{l}p= \\
0.097\end{array}$ \\
\hline Recurrence of bleeding episode & 0 & 0 & 1 & $<1$ & - \\
\hline Abdominal pain & 1 & $<1$ & 2 & $<1$ & - \\
\hline Hemoptysis & 0 & 0 & 1 & $<1$ & - \\
\hline Hypoglycemia episode following procedure & 0 & 0 & 1 & $<1$ & - \\
\hline Chest pain & 0 & 0 & 3 & $<1$ & - \\
\hline Febrile episode (also had chest pain) & 0 & 0 & 1 & $<1$ & - \\
\hline Headache, fatigue, dizziness & 1 & $<1$ & 0 & 0 & - \\
\hline Sore throat & 1 & $<1$ & 0 & 0 & - \\
\hline
\end{tabular}

Of note, patients who underwent primary prophylaxis had a lower mean number of endoscopy throughout their treatment course (3.897 vs $6.269, p=0.001$, Table 1 ). Few patients in either group achieved complete eradication of varices at their final endoscopy without significant difference in either group ( $7 \%$ vs $15 \%$ of 
patients, $p=0.188$ ); however, primary prophylaxis had a greater proportion of absent or Grade 1 varices at the time of endoscopy (Tables 1 and 2 ) and some patients were still actively undergoing therapy at the time this study was created. There were $18(21 \%)$ patients in the primary prophylaxis group who eventually had an episode of gastrointestinal bleeding during their course. Patient characteristics of those who had breakthrough bleeding are summarized on Table 3. Interestingly, 7 (39\%) of the patients with breakthrough bleeding had non-variceal causes of bleeding indentified at the time of endoscopy. However, these cases were still considered breakthrough bleeding as variceal bleeding could not be definitively ruled out. That said, patients in the primary prophylaxis group underwent fewer portosystemic shunts ( $6 \%$ vs $35 \%$ of patients, $p<0.001)$ with no significant differences in transplant $(24 \%$ vs $27 \%$ of patients, $p=0.0819$ ) or death ( $5 \%$ vs $13 \%$ of patients, $p=0.061$ ). Kaplan-Meier analysis showed that patients in the primary prophylaxis group had better overall survival (Fig. 1a, log-rank $p=0.024$ ) and survival without portosystemic shunt or LT (Fig. 1b, log-rank $p<0.001$ ) than those in the secondary prophylaxis group. Recorded deaths were not due to variceal bleeding or the endoscopic procedures themselves. 
Table 3

Characteristics of those with breakthrough bleeding despite primary prophylaxis

\begin{tabular}{|c|c|c|}
\hline & $\mathbf{n}$ & $\%$ \\
\hline Total Patients & 18 & 100 \\
\hline Underlying Diagnosis & - & - \\
\hline Alpha-1-antitrypsin Deficiency & 1 & 6 \\
\hline Autoimmune Hepatitis & 2 & 11 \\
\hline Biliary Atresia & 3 & 17 \\
\hline Cavernous Transformation / Thrombosis of the Portal Vein & 1 & 6 \\
\hline Congenital Hepatic Fibrosis & 1 & 6 \\
\hline Cystic Fibrosis Related Liver Disease & 4 & 22 \\
\hline Primary Sclerosing Cholangitis & 3 & 17 \\
\hline Other & 3 & 17 \\
\hline Mean age at first endoscopy (SD) & $8.39(5.61)$ & - \\
\hline Age range at first endoscopy & $\begin{array}{l}0.76- \\
17.71\end{array}$ & \\
\hline Variceal grade prior to breakthrough bleeding & - & - \\
\hline None & 2 & 11 \\
\hline Grade 1 / small / mild & 10 & 56 \\
\hline Grade 2 / medium / moderate & 1 & 6 \\
\hline Grade 3 / large / severe & 5 & 28 \\
\hline $\begin{array}{l}\text { Patient received ligation / sclerotherapy in endoscopy prior to breakthrough } \\
\text { bleeding }\end{array}$ & 3 & 17 \\
\hline Presenting symptom & - & - \\
\hline Hematemesis & 3 & 17 \\
\hline Melena / bloody stools & 10 & 56 \\
\hline Anemia & 1 & 6 \\
\hline Hemoptysis & 1 & 6 \\
\hline Other & 3 & 17 \\
\hline Variceal grade at the time of breakthrough bleeding & - & - \\
\hline
\end{tabular}




\begin{tabular}{|c|c|c|}
\hline & $\mathrm{n}$ & $\%$ \\
\hline None & 2 & 11 \\
\hline Grade 1 / small / mild & 6 & 33 \\
\hline Grade 2 / medium / moderate & 3 & 17 \\
\hline Grade 3 / large / severe & 6 & 33 \\
\hline Visualized but not graded & 1 & 6 \\
\hline Nonvariceal cause of bleeding identified & 7 & 39 \\
\hline Ulcer or gastritis & 4 & 22 \\
\hline Anal fissure & 1 & 6 \\
\hline Inflammatory bowel disease & 1 & 6 \\
\hline H. pylori & 1 & 6 \\
\hline Treatment at the time of bleeding & - & - \\
\hline None & 7 & 39 \\
\hline Secondary ligation & 10 & 6 \\
\hline Secondary sclerotherapy & 1 & 6 \\
\hline Mean years from first endoscopy to breakthrough bleeding (SD) & $2.25(2.28)$ & - \\
\hline Range of years from first endoscopy to breakthrough bleeding & $0.11-7.56$ & - \\
\hline Mean years from previous endoscopy to breakthrough bleeding (SD) & $0.70(0.64)$ & - \\
\hline Range of years from previous endoscopy to breakthrough bleeding & $0.04-2.35$ & - \\
\hline Outcomes & - & - \\
\hline Portosystemic shunt & 2 & 11 \\
\hline Liver transplant & 6 & 33 \\
\hline Death & 3 & 17 \\
\hline Shunt and transplant free survival & 7 & 39 \\
\hline
\end{tabular}

\section{Discussion}

Gastroesophageal varices develop as the PSG rises to $>10 \mathrm{mmHg}$ in adults and a PSG $>12 \mathrm{mmHg}$ is predictive of those adults who will develop variceal hemorrhage, although pediatric confirmatory data are lacking and children may develop complications at lower gradients than adults ${ }^{3,9,10}$. The true incidence and prevalence of PHTN and variceal hemorrhage in pediatric patients is undereported. Current data are 
often limited to disease specific populations or registries. One study in long term survivors of biliary atresia without LT revealed up to two thirds of patients had PHTN and approximately $20 \%$ developed bleeding from esophageal varices ${ }^{11}$. The treatment of variceal hemorrhage is consistent across ages and includes pharmacologic measures (such as vasopressin or somatostatin) as well as endoscopic measures (such as EVL and sclerotherapy when EVL is not feasible). Refractory cases can be managed emergently with the Sengstaken-Blackmore tube. Shunt procedures (either transjugular intrahepatic portosystemic shunts or surgical) and transplantation are offered to the stabilized patient who has recurrent variceal hemorrhage not amenable to the above measures ${ }^{3}$.

Despite its routine use in adults, the primary prevention of gastrointestinal bleeding from varices remains underreported in pediatrics. Adult guidelines clearly recommend endoscopy in a patient with PHTN as the presence of varices can help determine prophylactic measures including NSBB and EVL ${ }^{2}$. These measures, have been shown to improve morbidity and mortality from variceal hemorrhage in adult patients with PHTN with no synergetic effect though and repeat endoscopy is recommended with intervals dependent on the progression of liver disease ${ }^{12}$.

Unfortunately, there is a paucity of data assessing the safety, efficacy, and outcomes of primary prophylaxis in pediatric PHTN; this includes studies on the use of both NSBB and endoscopy. As a result, there have been calls for further research acknowledging the difficulties of applying these results widely 4 . Guidelines, such as the Baveno VI Pediatric Satellite Symposium, have addressed this clinical question with a "do no harm" approach until further research can be completed ${ }^{13}$. However, this recommendation requires discussion as institutions balance their patient populations with local expertise and available resources in an event that can have 6 week mortality as high as $8.8 \%^{8}$. While there are no published multicentered, randomized, and prospective studies, there is a growing body of evidence that endoscopic primary prophylaxis may be safe and effective in pediatric portal hypertension.

In 2013, the French group Duché et al reported their experience in primary and secondary prophylaxis in biliary atresia ${ }^{6}$. They found that primary prophylaxis was well tolerated and recommended continued surveillance even after eradication of varices in biliary atresia. This was then followed in 2017 by a retrospective study by Duché et al for all causes of portal hypertension ${ }^{14}$. In this study they identified high risk varices (grade 3 , grade 2 with red wale markings, and gastric varices) and noted that prevention of first bleed appeared to decrease morbidity and mortality. Additionally, Pimenta JR et al found endoscopic primary prophylaxis to be superior to NSBB in a small study of 26 children with cirrhosis in its ability to reduce bleeding events ${ }^{15}$. Kang KS et al found that EVL used to prevent rebleeding was safe and effective $^{16}$. This is especially relevant as recent studies have shown that non-invasive measures have difficulty predicting gastrointestinal bleeding. Lampela et al. demonstrated that liver biochemistry labwork, liver stiffness, and predictive scores had difficulty predicting the presence of varices in pediatric patients with biliary atresia with similar conclusions by Angelico R et al ${ }^{17,18}$. 
Pediaatric guidelines provide a caveat that patients without easy access to appropriate levels of medical care may benefit from primary prophylaxis given the potential of rapid large volume hemorrhage ${ }^{19}$. Our practice historically established endoscopy for primary and secondary prophylaxis due to our significant rural patient population. This has led to a large pediatric population who have undergone both primary and secondary endoscopy prophylaxis with reportable clinical longitudinal data, outcomes, and complication rates to describe over a long period of time (Fig. 1a and 1b).

Most importantly, we report little complications associated with prophylactic endoscopy (Table 2). The majority of complications resolved with supportive care and only 1 case required significant intervention (a single balloon dilation for a stricture). While 18 (21\%) patients of the primary prophylaxis group had breakthrough bleeding, 7 (39\%) of those cases had non-variceal causes of bleeding identified at the time of endoscopy and only 6 (33\%) had large varices identified at the time of bleeding. These results suggest that prophylactic endoscopy in pediatric portal hypertension may be a safe alterantive to the watchful waiting approach.

There were significant differences and similarities noted between the primary and secondary prophylactic groups. However, it is important to note that our study was not designed to evaluate whether the differences were due to treatment strategy (primary vs second prophylaxis) or other confounding factors such as inherent patient characteristics. Neither group had significant differences in age of initial presentation or diagnoses other than autoimmune hepatitis and cavernous transformation of the portal vein / portal vein thrombosis (Table 1). Initial MELD score did not show significant differences between the two groups while initial PELD scores revealed a statistically significant difference in means and a higher maximum initial PELD score in the secondary prophylaxis group. This may suggest that some young patients in the secondary group had more advanced liver disease at presentation. However, limitations to this interpretation include a debatable clinical significance of a mean PELD of 1.92 vs 6.10, that PELD is not a prognostic indicator of variceal bleeding, and the low numbers of available PELD and MELD scores in our patients that could skew results. Our primary prophylaxis group experienced a significantly decreased mean number of total endoscopies, usage of NSBB, PICU admissions, portosystemic shunts and higher rates of shunt and transplant free survival without significant complications. Again, it remains unclear if this is due to treatment strategy or patient characteristics (such as severity of disease) and should be further studied.

Our study's strength is longtidunal safety and efficacy over a long period of time that can add to the growing body of evidence that prophylactic endoscopy should be further studied in pediatric patients with portal hypertension to determine best practices. Limitations to this study include its retrospective nature and that patients were not randomized into either group. Importantly, there is also no control group who did not receive any prophylactic endoscopy. Our center does not a have large enough group of patients with portal hypertension who did not receive any prophylactic endoscopy to meaningfully study. While our analysis can describe safety and outcomes of pediatric prophylactic endoscopy, alone it is unable to fully address the debate between prophylactic endoscopy compared to watchful waiting. Additionally, our patient population reflects a wide etiology of PHTN and recommendations for specific diseases are not 
easily made. There may be disease specific factors that could effect outcomes. However, comparing groups by disease resulted in too small of sample sizes to draw meaningful conclusions. Our results also may better reflect our patient pool and local expertise rather than generalizable practice.

Further studies should be performed to clearly delineate morbidity and mortality between prophylactic endoscopy of either type and watchful waiting in the pediatric setting across a spectrum of disease. Ideally, these would be prospective and multicenter given the lower volumes of patients in pediatric centers compared to adult practices. Large scale multicentre patient registries can also contirubute further to our knowledge and understanding of PHTN and its management in children. However, this study provides supporting longitudinal evidence that prophylactic endoscopy can be considered a reasonably safe protocol to manage pediatric PHTN.

\section{Abbreviations}

EVL - endoscopic variceal ligation, LT - liver transplant, MELD - Model for End-Stage Liver Disease, NSBB - non-selective beta blockers, PELD - Pediatric End-Stage Liver Disease, PICU - pediatric intensive care unit, PHTN - portal hypertension, PSG - portosystemic gradient

\section{Declarations}

\section{Acknowledgements:}

The authors would like to acknowledge and thank Mark Connelly PhD for his assistance in statistical analysis as well as James F. Daniel MD for establishing the Liver Care Center at Children's Mercy Kansas City with this institutional protocol

\section{Author Contributions:}

Study conception and design - VS, JFD, TG; data acquisition - VS, AB, HW; analysis and data interpretation - VS, RTF, JFD, TG; drafting of the manuscript - VS; critical revisions: VS, AB, HW, RTF, JFD, TG

\section{Competing Interests and Sources of Funding:}

This research did not receive any specific grant from funding agencies in the public, commercial, or notfor-profit sectors and the authors declare no competing interests

\section{References}

1. Beppu K, Inokuchi K, Koyanagi N, Nakayama S, et al. Prediction of variceal hemorrhage by esophageal endoscopy. Gastrointest Endosc. 1981 Nov;27(4):213-8

2. Garcia-Tsao G, Abraldes JG, Berzigotti A, Bosch J. Portal hypertensive bleeding in cirrhosis: Risk stratification, diagnosis, and management: 2016 practice guidance by the American Association for 
the study of liver diseases. Hepatology. 2017 Jan;65(1):310-335

3. Shneider BL, Bosch J, de Franchis R, et al; expert panel of the Children's Hospital of Pittsburgh of UPMC. Portal hypertension in children: expert pediatric opinion on the report of the Baveno $\mathrm{V}$ Consensus Workshop on Methodology of Diagnosis and Therapy in Portal Hypertension. Pediatr Transplant. 2012;16(05):426-437

4. Ling SC, Walters T, McKiernan PJ, Schwarz KB, et al. Primary prophylaxis of variceal hemorrhage in children with portal hypertension: a framework for future research. J Pediatr Gastroenterol Nutr. 2011 Mar;52(3):254-61

5. Molleston JP, BL Shneider. Preventing Variceal Bleeding in Infants and Children: Is Less More? Gastroenterology. 2013 Oct;145(4):719-22

6. Duché M, Ducot Bm Ackermann O, Baujard C, Chevret L, et al. Experience with endoscopic management of high-risk gastroesophageal varices, with and without bleeding, in children with biliary atresia. Gastroenterology. 2013 Oct;145(4):801-7

7. Philips CA, Sahney A. Oesophageal and gastric varices: historical aspects, classifications and grading: everything in one place. Gastroenterol Rep (Oxf). 2016 Aug;4(3):186-195

8. Molleston JP, Bennett WE Jr. Mortality, Risk Factors and Disparities Associated with Esophageal Variceal Bleeding in Children's Hospitals in the US. J Pediatr. 2021 Jan 12:S0022-3476(21)00005-6. doi: 10.1016/j.jpeds.2020.12.082. Epub ahead of print. PMID: 33450222.

9. Lake-Bakaar G, Ahmed M, Evenson A, Bonder A, et al. Hagen-Poiseuille's law: The link between cirrhosis, liver stiffness, portal hypertension and hepatic decompensation. World J Hepatol. 2015 Jan 27;7(1):28-32

10. Slowik V, Monroe EJ, Friedman S, Hsu EK, Horslen S. Pressure gradients, laboratory changes, and outcomes with transjugular intrahepatic portosystemic shunts in pediatric portal hypertension. Pediatric Transplantation. 2019;e13387. doi: 10.1111/petr.13387

11. Schneider BL, Abel B, Haber B, Karpen SJ, et al. Portal hypertension in children and young adults with biliary atresia. J Pediatr Gastroenterol Nutr. 2012 Nov;55(5):567-73

12. de Franchis R, Baveno VI Faculty. Expanding consensus in portal hypertension: Report of the Baveno VI Consensus Workshop: Stratifying risk and individualizing care for portal hypertension. J Hepatol. 2015 Sep;63(3):743-52

13. Shneider BL, de Ville de Goyet, Leung DH, Srivastava A, et al. Primary prophylaxis of variceal bleeding in children and the role of MesoRex Bypass: Summary of the Baveno VI Pediatric Symposium. Hepatology. 2016 Apr;63(4):1368-80. Doi: 10.1002/hep.28153. Epub 2015 Oct 23.

14. Duché $M$, Ducot $B$, Ackermann $O$, Guérin F, et al. Portal hypertension in children: High-risk varices, primary prophylaxis and consequences of bleeding. J Hepatol. 2017 Feb;66(2):320-327

15. Pimenta JR, Ferreira AR, Bittencourt PF, Resende CB, et al. Evaluation of primary prophylaxis with propranolol and elastic band ligation in variceal bleeding in cirrhotic children and adolescents. Arg Gastroenterol. 2016 Oct-Dec;53(4):257-261. Doi: 10.1590/S0004-28032016000400009 
16. Kang KS, Yang HR, Ko JS, Seo JK. Long-term Outcomes of Endoscopic Variceal Ligation to Prevent Rebleeding in Children with Esophageal Varices. J Korean Med Sci. 2013 Nov; 28(11): 1657-1660

17. Lampela H, Hukkinen M, Kosola S, Jahnukainen T, Pakarinen MP. Poor performance of noninvasive predictors of esophageal varices during primary prophylaxis surveillance in biliary atresia. J Pediatr Surg. 2020 Jun 20:S0022-3468(20)30437-1. doi: 10.1016/j.jpedsurg.2020.06.017. Epub ahead of print. PMID: 32682540.

18. Angelico R, Pietrobattista A, Candusso M, Tomarchio S, Pellicciaro M, Liccardo D, Basso MS, Grimaldi C, Saffioti MC, Torroni F, Dall'Oglio L, Torre G, Spada M. Primary Prophylaxis for Gastrointestinal Bleeding in Children With Biliary Atresia and Portal Hypertension Candidates for Liver Transplantation: A Single-Center Experience. Transplant Proc. 2019 Jan-Feb;51(1):171-178. doi: 10.1016/j.transproceed.2018.04.074. Epub 2018 Jun 28. PMID: 30655149.

19. Gugig R, Rosenthal P. Management of portal hypertension in children. World J Gastroenterol. 2012 Mar 21; 18(11):1176-1184

\section{Figures}
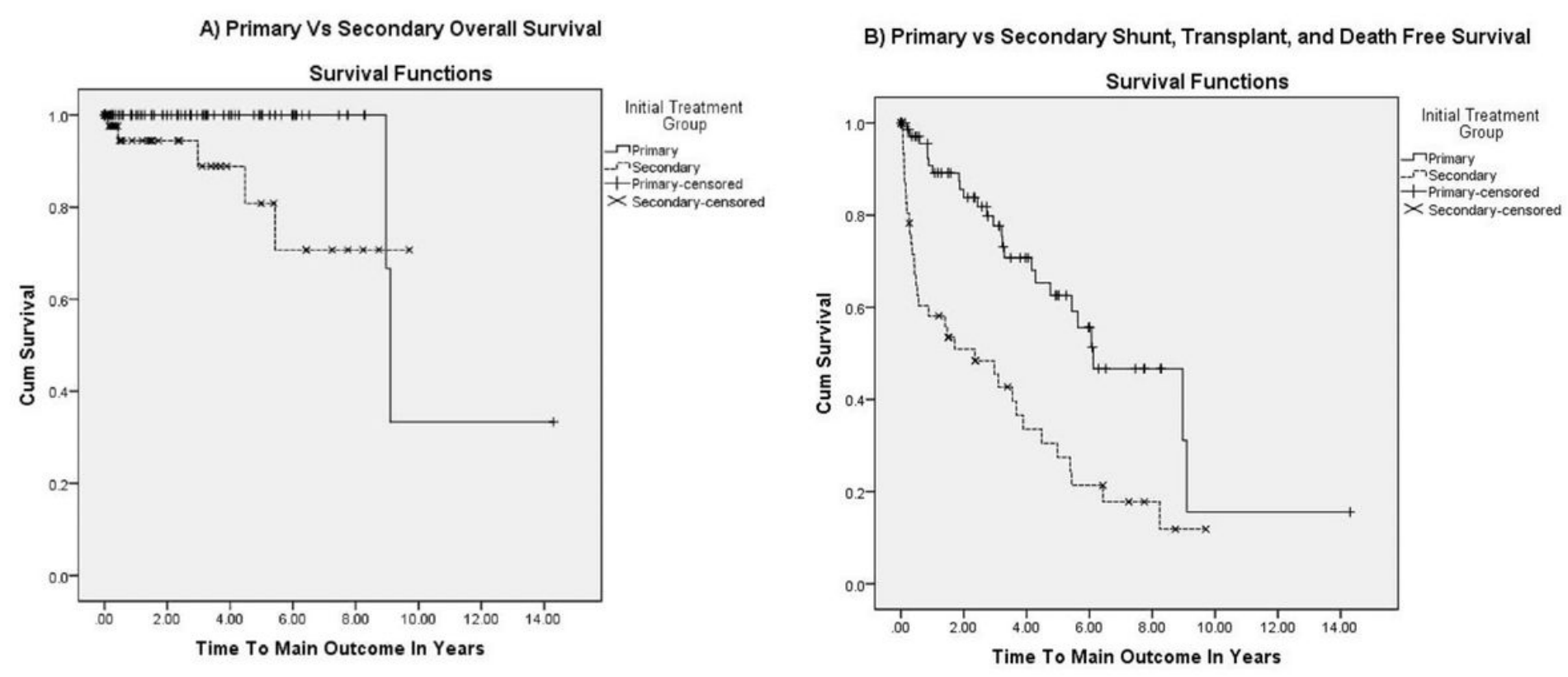

\section{Figure 1}

(a) Kaplan-Meier Overall Survival Curve for Primary and Secondary Endoscopic Prophylaxis (log-rank $\mathrm{p}=$ 0.024). (b) Kaplan-Meier Survival Curve without portosystemic shunt or liver transplant for Primary and Secondary Endoscopic Prophylaxis (log-rank $p=0.024)$ 